\title{
Avaliação do conhecimento e capacitação dos agentes comunitários de saúde a respeito da escala de depressão geriátrica
}

\author{
Knowledge assessment and training of community health agents regarding the geriatric \\ depression scale
}

Evaluación del conocimiento y capacitación de los agentes de salud de la comunidad sobre la escala de depresión geriátrica

Ângela Milhomem Vasconcelos ${ }^{1}$, Julia Medeiros Santana ${ }^{1}$, Maria Clara Souza Fernandes ${ }^{1}$, Pâmela Gabrielle Lima Barreiros ${ }^{1}$, Yana de Medeiros Souza Lima ${ }^{1 *}$, Bruno Rodrigues Salvador ${ }^{1}$, Karine da Silva Ribeiro Ramos ${ }^{1}$, Mateus de Souza Castro ${ }^{1}$, Santino Carvalho Franco ${ }^{1}$, José Antônio Cordero da Silva'.

\section{RESUMO}

Objetivo: Capacitar os Agentes Comunitários de Saúde (ACS) para aplicabilidade da Escala de Depressão Geriátrica (GDS). Métodos: Estudo do tipo coorte, prospectivo, longitudinal e quantitativo. Participaram 12 ACS, responsáveis pela cobertura da área adstrita da Unidade de Saúde da Família (USF). Consideraram-se elegíveis para a pesquisa os ACS cadastrados que aceitaram participar da pesquisa. Resultados: Apenas $16,60 \%$ dos ACS possuíam conhecimento sobre a GDS antes da apresentação e após explicação, todos demonstraram ter conhecimento. Antes da capacitação, 8,30\% dos ACS demonstraram conhecer as formas de apresentação da mesma, porém, após, $100 \%$ afirmou ter domínio sobre as formas de apresentação da escala. Sobre o conteúdo, nenhum dos ACS demonstrou saber quais eram e, após a capacitação, $75 \%$ dos pesquisados afirmaram ter conhecimento sobre as perguntas em questão, enquanto $25 \%$ afirmou ainda falta de domínio. Sobre a aplicação da GDS, nenhum dos ACS possuía conhecimento e após a explanação, $91,70 \%$ demonstrou domínio da aplicação e 8,30\% ainda se apresentaram inseguros nesse quesito. Conclusão: É válido a utilização de métodos que possibilitem aos ACS atuarem como elo entre saúde e comunidade. Estratégias de avaliação rápida, como a GDS, permitem examinar uma grande parcela da população-alvo, facilitando a referência para os serviços médicos existentes.

Palavras-chave: Depressão, Idoso, Serviços de saúde para idosos.

\section{ABSTRACT}

Objective: To train Community Health Agents (SCA) to apply the Geriatric Depression Scale (GDS). Methods: Cohort, prospective, longitudinal and quantitative study. Twelve ACS participated, responsible for covering the assigned area of the Family Health Unit (USF). Registered SCAs who agreed to participate in the research were considered eligible for the research. Results: Only $16.60 \%$ of the SCA had knowledge about the GDS before the presentation and after explanation, all demonstrated knowledge. Before the training, $8.30 \%$ of the SCA demonstrated to know the ways of presenting it, however, after, $100 \%$ claimed to have mastery over the ways of presenting the scale. Regarding the content, none of the SCA demonstrated to know what they were and, after training, $75 \%$ of the respondents said they had knowledge about the questions in question, while $25 \%$ said they still lack mastery. Regarding the application of the GDS, none of the SCA had knowledge and after the explanation, $91.70 \%$ demonstrated mastery of the application and $8.30 \%$ were still unsafe in this regard. Conclusion: It is valid to use methods that enable SCAs to act as a link between health and the community. Rapid assessment strategies, such as the GDS, allow examining a large portion of the target population, facilitating referral to existing medical services.

Keywords: Depression, Seniors, Health services for the elderly.

${ }^{1}$ Centro Universitário Metropolitano da Amazônia (UNIFAMAZ), Belém - PA.

*E-mail: yanamedeiros25@gmail.com 


\section{RESUMEN}

Objetivo: capacitar a Agentes de Salud Comunitaria (ACS) para aplicar la Escala de Depresión Geriátrica (GDS). Métodos: estudio de cohorte, prospectivo, longitudinal y cuantitativo. Participaron 12 ACS, responsables de cubrir el área asignada de la Unidad de Salud Familiar (USF). Los ACS registrados que aceptaron participar en la investigación se consideraron elegibles para la investigación. Resultados: solo el $16.60 \%$ de la ACS tenía conocimiento sobre el GDS antes de la presentación y después de la explicación, todos demostraron conocimiento. Antes del entrenamiento, el $8.30 \%$ de la ACS demostró conocer las formas de presentarlo, sin embargo, después, el 100\% afirmó tener dominio sobre las formas de presentar la escala. Con respecto al contenido, ninguno de los ACS demostró saber lo que eran y, después de la capacitación, el $75 \%$ de los encuestados dijeron que tenían conocimiento sobre las preguntas en cuestión, mientras que el $25 \%$ dijo que todavía les falta dominio. Con respecto a la aplicación del GDS, ninguno de los ACS tenía conocimiento y después de la explicación, el $91.70 \%$ demostró el dominio de la aplicación y el $8.30 \%$ aún no era seguro a este respecto. Conclusión: es válido utilizar métodos que permitan a los ACS actuar como un vínculo entre la salud y la comunidad. Las estrategias de evaluación rápida, como el GDS, permiten examinar una gran parte de la población objetivo, facilitando la derivación a los servicios médicos existentes.

Palabras clave: Depresión; Anciano; Servicios de salud para personas mayores.

\section{INTRODUÇÃO}

Os Agentes Comunitários de Saúde (ACS) constituem uma categoria profissional numerosa e amplamente capilarizada no território brasileiro, atuando a partir de 1991 na Atenção Básica em Saúde no âmbito do Sistema Único de Saúde (SUS), desde sua inserção no Programa de Agentes Comunitários de Saúde (PACS), depois nas equipes do Programa de Saúde da Família (PSF) e, atualmente, integrados na chamada Estratégia Saúde da Família (ESF). O ACS, de acordo com a Portaria №. 648, de 28 de março de 2006, é o elo entre a equipe de saúde e a população, sendo um profissional importante na ESF (BORTOLI FR, et al., 2017; KNOCHENHAUER CCLS e VIANNA KMP, 2016).

No âmbito do SUS, as atividades dos ACS têm se modificado, seu trabalho tem se articulado ao de outros profissionais integrantes da ESF e suas funções foram reconhecidas, desde 2002, como atividades profissionais, com o reconhecimento legal da profissão por meio da Lei 10.507. Eles são fundamentais em virtude da facilidade do acesso aos usuários no território de atuação, o que Ihes possibilita a identificação precoce de situações de risco e se configuram como um importante ator no fortalecimento do Sistema Único de Saúde (SUS) (GOMES RCM e SOUZA CD, et al., 2015).

A esses profissionais cabe reunir informações de saúde sobre a comunidade e realizar visitas domiciliares na área adstrita, produzindo dados capazes de dimensionar os principais problemas de saúde da sua comunidade considerando os critérios de risco e vulnerabilidade, além de cadastrar todas as pessoas do território, orientando as famílias quanto à utilização dos serviços de saúde disponíveis, desenvolver atividades de promoção, prevenção de doenças e agravos e de vigilância em saúde. (GOMES RCM, et al., 2015; FIGUEIREDO EM, 2015).

Em vista disso, é notória a existência da associação entre doenças crônicas sistêmicas e transtornos psiquiátricos, percebida em uma parcela relevante de pacientes tratados na atenção básica, o que pode acabar camuflando os sintomas psiquiátricos e prejudicando assim, o diagnóstico. Em idosos, isso é ainda mais comum, o que dificulta ainda mais o diagnóstico da depressão, considerando-se que as doenças crônicas sistêmicas são mais constantes e as queixas mais exacerbadas, tornando os sintomas depressivos menos aparentes, e muitas vezes subdiagnósticados. (SCHAAB BL, et al., 2017).

No que se refere à saúde mental, a depressão é o transtorno de humor mais frequente entre os idosos. A depressão maior é caracterizada por humor deprimido, perda do interesse ou prazer, alterações do funcionamento biológico, com repercussões importantes na vida do indivíduo e com duração, sem tratamento, de meses a anos. Recente metanálise brasileira aponta para estudos com altas taxas de prevalência de sintomas depressivos nessa população, com proporções que variam entre 20 e $56 \%$ em 
pacientes de acompanhamento ambulatorial, 23 a 42\% em indivíduos hospitalizados e de 11 até $65 \%$ em idosos institucionalizados. (SANTOS-ORLANDI AA, et al., 2017; LIMA AMP, et al., 2016).

Segundo a Organização das Nações Unidas (ONU), os idosos representarão um quarto da população mundial projetada, ou seja, cerca de 2 bilhões de indivíduos, vivenciando uma rápida e intensa transição demográfica e epidemiológica. (SILVA AR, et al., 2017; GONZÁLEZ ACT, et al., 2016). No Brasil, a representatividade desta faixa etária corresponde a $8,6 \%$ do total da população do país (14,5 milhões), com projeção para 9,7\% em 2050. Em detrimento disso, ocorre uma maior demanda por serviços de saúde em decorrência das patologias relacionadas ao envelhecimento, entre as quais destacamos a depressão (RALDI GV, et al., 2016; SEMED DC, et al., 2016).

No Pará, foi evidenciado um contingente expressivo da população idosa com quadro de depressão, com predominância de idosos do sexo feminino e em idosos acima de 80 anos e institucionalizados. Foram verificados ainda, outros fatores de risco, como história de depressão pregressa, viuvez, renda baixa, insatisfação com o suporte social, isolamento social, ansiedade, falta de atividades sociais, nível educacional baixo e uso de medicação antidepressiva. (RALDI GV, et al., 2016; SEMED DC, et al., 2016).

A importância de identificar a depressão na terceira idade se dá porque além de serem tratáveis, se crônicas, são resultados de um tratamento inapropriado, e depressões severas são um grande problema de saúde pública. Por essa razão, acredita-se em iniciativas que investigam informações relevantes de saúde mental, por meio da busca ativa com baixo custo, como a Escala de Depressão Geriátrica (GDS), instrumento de avaliação para depressão, desenvolvido e validado em 1983. (PEDREIRA RBS, et al., 2016; NABBE P, et al., 2017; LAUDISIO A, et al., 2017; GALERA SC, et al., 2017).

De acordo com Moreira (2017), a GDS é um dos instrumentos mais frequentemente utilizados para o rastreamento de depressão em idosos. A escala original tem 30 itens e foi desenvolvida especialmente para o rastreamento dos transtornos de humor em idosos, com perguntas que evitam a esfera das queixas somáticas. A GDS com 15 itens é uma versão curta da escala original e foi elaborada a partir dos itens que mais fortemente se correlacionavam com o diagnóstico de depressão. Esses itens, em conjunto, mostraram boa acurácia diagnóstica, com sensibilidade, especificidade e confiabilidade adequadas. Entre as suas vantagens destacam-se: é composta por perguntas fáceis de serem entendidas; tem pequena variação nas possibilidades de respostas; pode ser auto-aplicada ou aplicada por um entrevistador treinado. Assim, a GDS se torna uma ferramenta a mais para identificação de idosos que possuam características depressivas e a partir da sua aplicação, o ACS pode orientar ao idoso sobre a necessidade de um melhor acompanhamento. (MOREIRA CS, 2017; ALMEIDA AM, et al., 2016).

Mediante a negligência com o reconhecimento dos casos de depressão geriátrica nas áreas subjacentes às ESF e levando em consideração os escassos diagnósticos precoces de depressão geriátrica, esse estudo subsidiou a elaboração de questionários direcionados aos ACS com o objetivo de mensurar seus conhecimentos à respeito da GDS e otimizar sua aplicabilidade, oferecendo maior eficácia ao processo de triagem desses pacientes para melhor manejo da evolução e prognóstico da patologia. E, ao reconhecer esta interação mais próxima entre os usuários da ESF e os ACS, esse trabalho tem o propósito de contribuir para diminuir as barreiras da triagem dos idosos com depressão, contribuir para o diagnóstico precoce, bem como seu tratamento e prognóstico interferindo positivamente na qualidade de vida deste idoso e sua família.

\section{MÉTODOS}

O estudo foi elaborado do tipo coorte, prospectivo, longitudinal e quantitativo e o modelo de estudo é analítico. Foi realizado na Unidade de Saúde da Família (USF) de um bairro de uma cidade paraense. Esta é composta por enfermeiros, técnicos de enfermagem, médicos, agentes comunitários de saúde e uma equipe de Núcleo de Apoio à Saúde da Família (NASF). O período da realização da coleta de dados compreendeu o mês de abril de 2019. Foram convidados a participar da pesquisa 12 ACS, os quais são responsáveis pela cobertura da área adstrita da USF do Panorama XXI. A coleta contemplou a participação dos 12 ACS, pois não houve perdas ou faltas na amostra estimada. 
Foram considerados elegíveis para a pesquisa os ACS cadastrados como colaboradores da cobertura da USF Panorama XXI e que aceitaram participar da pesquisa. Foram considerados inelegíveis os ACS que se recusaram a assinar o Termo de Consentimento Livre e Esclarecido (TCLE).

A referente pesquisa foi constituída das cinco etapas descritas a seguir:

1․ Etapa: Foi solicitado um levantamento do total de ACS que prestam serviço para a USF.

$2^{\mathrm{a}}$ Etapa: Os pesquisadores convidaram os ACS a participar voluntariamente da pesquisa através da assinatura do TCLE. A pesquisa se deu através da aplicação de um questionário aos ACS, com o intuito de evidenciar o conhecimento deles a respeito da GDS.

3a Etapa: Os pesquisadores coletaram os dados do questionário aplicado com os ACS e posteriormente fizeram a capacitação quanto à aplicação da GDS, para que os pacientes recebam encaminhamento para um serviço de Atenção Secundária, onde possam ser investigados sinais de depressão nestes.

4a Etapa: Os pesquisadores aplicaram novamente o questionário para evidenciar se a capacitação realizada foi eficaz para o conhecimento dos ACS.

5aㅡㄹ Etapa: Realizaram a nova coleta de dados do questionário aplicado aos ACS e buscaram opiniões deles a respeito da eficácia da capacitação.

A pesquisa foi realizada após a aprovação do Comitê de Ética (CAAE: 01795218.9.0000.5701) e do gestor local. Todos os sujeitos envolvidos foram consultados sobre sua participação na investigação, e esclarecidos com leitura e assinatura do TCLE, de acordo com que preconiza a Resolução 466/12 do CNS/MS, o Código de Nuremberg e a declaração de Helsinque. Os dados coletados estão armazenados e ficaram por cinco anos, sendo posteriormente descartados.

\section{RESULTADOS}

A intervenção foi realizada na USF Panorama XXI, na cidade de Belém-PA, e dividida em duas etapas. $O$ primeiro momento ocorreu durante a manhã do dia 03 (três) de Abril de 2019, no qual foi aplicado o questionário, elaborado pelos pesquisadores, com o intuito de identificar o nível de conhecimento dos ACS a respeito da GDS e logo em seguida foi realizada a capacitação e uma pequena simulação afim de aplicar de maneira prática o tema abordado.

O segundo momento ocorreu, também pela parte da manhã, no dia 10 (dez) de Abril de 2019, no qual foi aplicado novamente o questionário elaborado pelos pesquisadores, dessa vez, com o intuito de evidenciar se a capacitação realizada foi eficaz para o conhecimento dos ACS abordados e se, de alguma forma, esse novo conhecimento contribuiu para suas ações no dia a dia, com a população idosa.

Participaram da pesquisa 12 ACS adscritos na área do Panorama XXI. A partir dos dados obtidos no gráfico 1, verificou-se que apenas $16,60 \%$ dos ACS tinham conhecimento a respeito da GDS antes da apresentação desta, enquanto os que não a conheciam totalizaram $83,40 \%$. Após a exposição sobre a escala, todos demonstraram ter conhecimento.

Antes da capacitação sobre a aplicação da GDS, 8,30\% dos ACS demonstraram conhecer a forma de apresentação da mesma, enquanto $91,70 \%$ afirmaram que não e, após a conversa expositiva, 100\% afirmou ter domínio sobre a forma de apresentação de tal escala.

Sobre as perguntas que compõem a escala, antes da palestra, nenhum dos ACS demonstrou saber quais eram essas perguntas e, após a capacitação realizada pelos pesquisadores, $75 \%$ dos pesquisados afirmaram ter conhecimento sobre as perguntas em questão e $25 \%$ afirmou que ainda não havia obtido este domínio.

Quando foram questionados sobre a aplicação da GDS, nenhum dos ACS afirmou ter conhecimento a respeito, após a explanação dos pesquisadores, $91,70 \%$ demonstrou ter domínio sobre a aplicação e 8,30\% ainda se apresentaram inseguros nesse quesito (Gráfico 1). 
Gráfico 1 - Comparação das respostas acerca do conhecimento da GDS pelos ACS, antes e depois da capacitação, na USF Panorama XXI, Belém - PA, Brasil, 2019

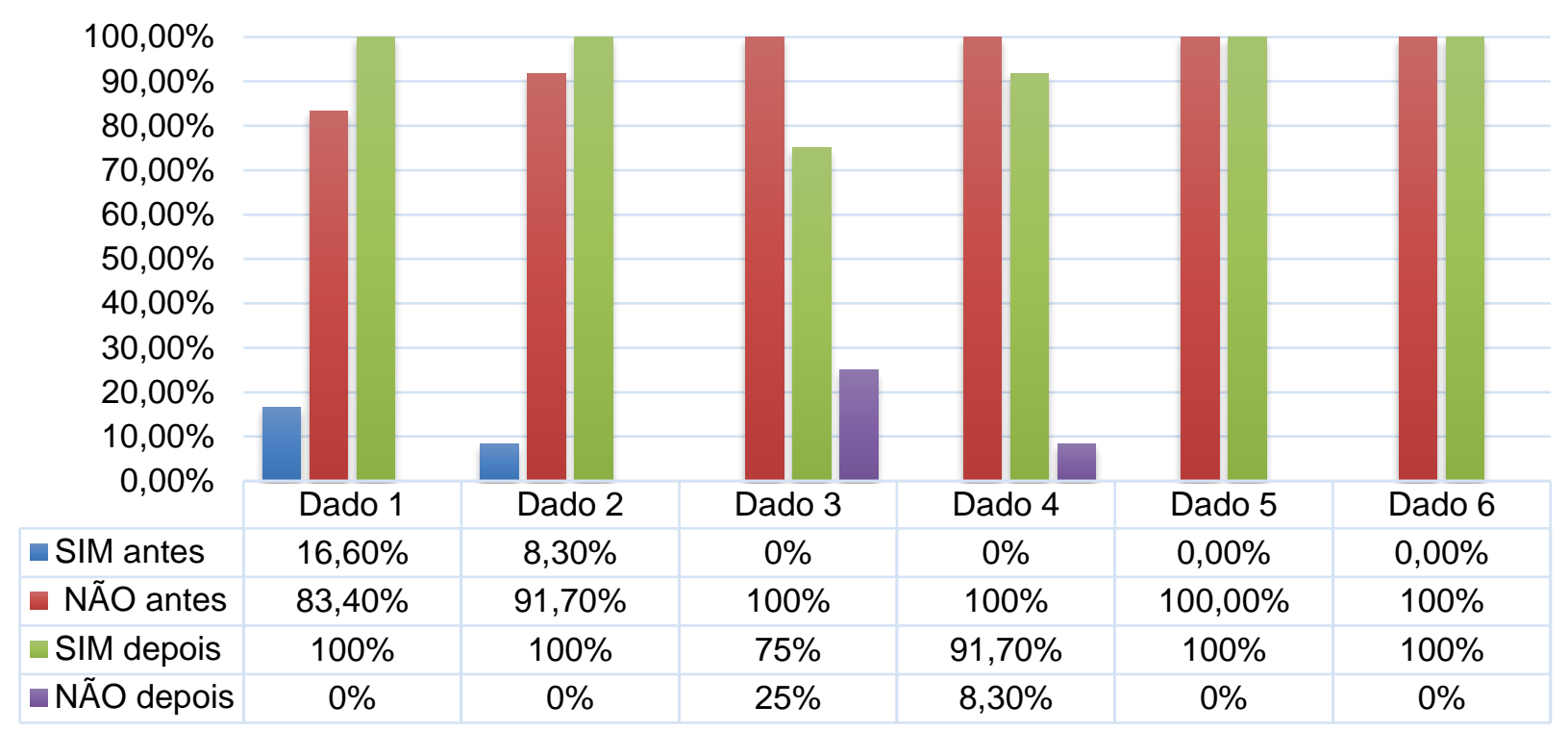

\section{Legenda:}

Dado 1: Conhecimento do ACS acerca da existência da GDS;

Dado 2: Conhecimento do ACS em relação as formas de apresentação da GDS;

Dado 3: Conhecimento do ACS acerca das perguntas que compõem a GDS;

Dado 4: Aplicação da escala pelo ACS;

Dado 5: Conhecimento do ACS acerca da finalidade da utilização da GDS;

Dado 6: Conhecimento do ACS acerca dos profissionais capacitados para aplicação da GDS.

Fonte: Vasconcelos AM, et al., 2020.

No primeiro momento da pesquisa, fora questionado aos ACS sobre o conhecimento destes a respeito da efetividade e importância da GDS no que tange a triagem de sintomas depressivos em idosos e, após a capacitação, o mesmo questionamento fora realizado, obtendo-se respostas completamente distintas entre si. Antes da capacitação, nenhum dos ACS afirmou ter conhecimento sobre a efetividade da escala, enquanto que, após a intervenção, todos demonstraram conhecer essa efetividade (Gráfico 2).

Gráfico 2 - Comparação das repostas acerca do conhecimento sobre a efetividade da escala para triagem dos sintomas depressivos nos idosos, pelos ACS, antes e depois da capacitação, na USF Panorama XXI, Belém - PA, Brasil, 2019.

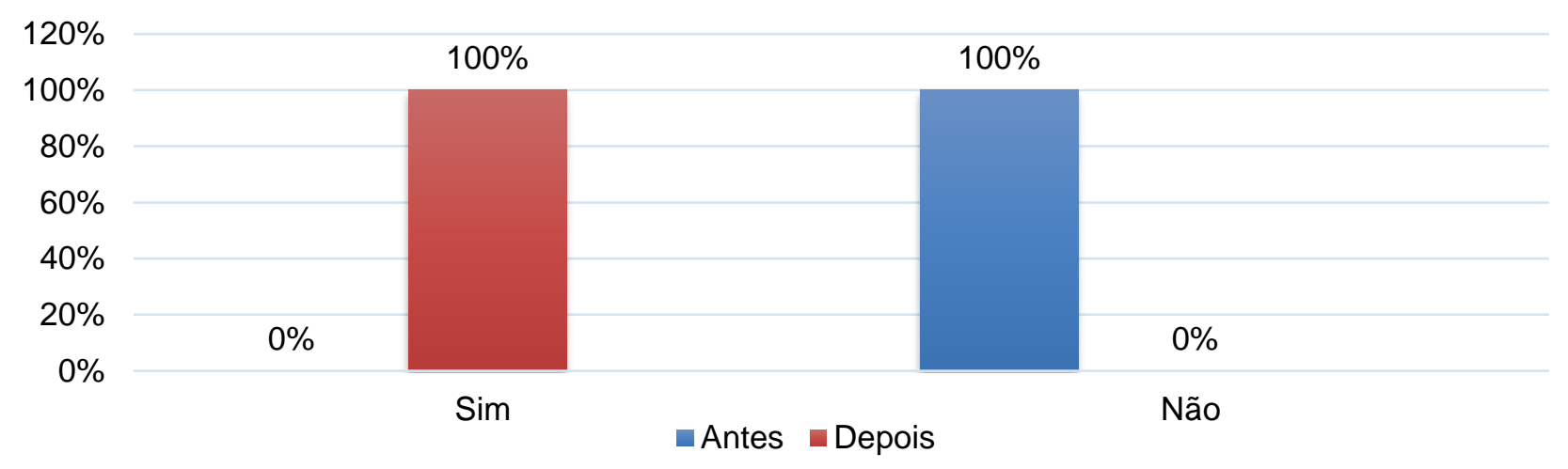

Fonte: Vasconcelos AM, et al., 2020. 
Quando questionado aos ACS se esses tinham domínio sobre a forma de apresentação dos sintomas depressivos nos idosos, apenas $33,30 \%$ demonstraram conhecimento sobre os sintomas e $66,70 \%$ afirmaram o contrário. Logo após a capacitação, o mesmo questionamento fora feito, obtendo-se $83,30 \%$ de respostas afirmativas para o reconhecimento sintomático da depressão em idosos, enquanto $16,70 \%$ dos ACS afirmaram que não possuir esse conhecimento (Gráfico 3).

Gráfico 3 - Comparação das respostas acerca da identificação de sintomas depressivos nos idosos nas áreas de abrangência respectiva a cada ACS, antes e depois da capacitação, na USF Panorama XXI, Belém - PA, Brasil, 2019.

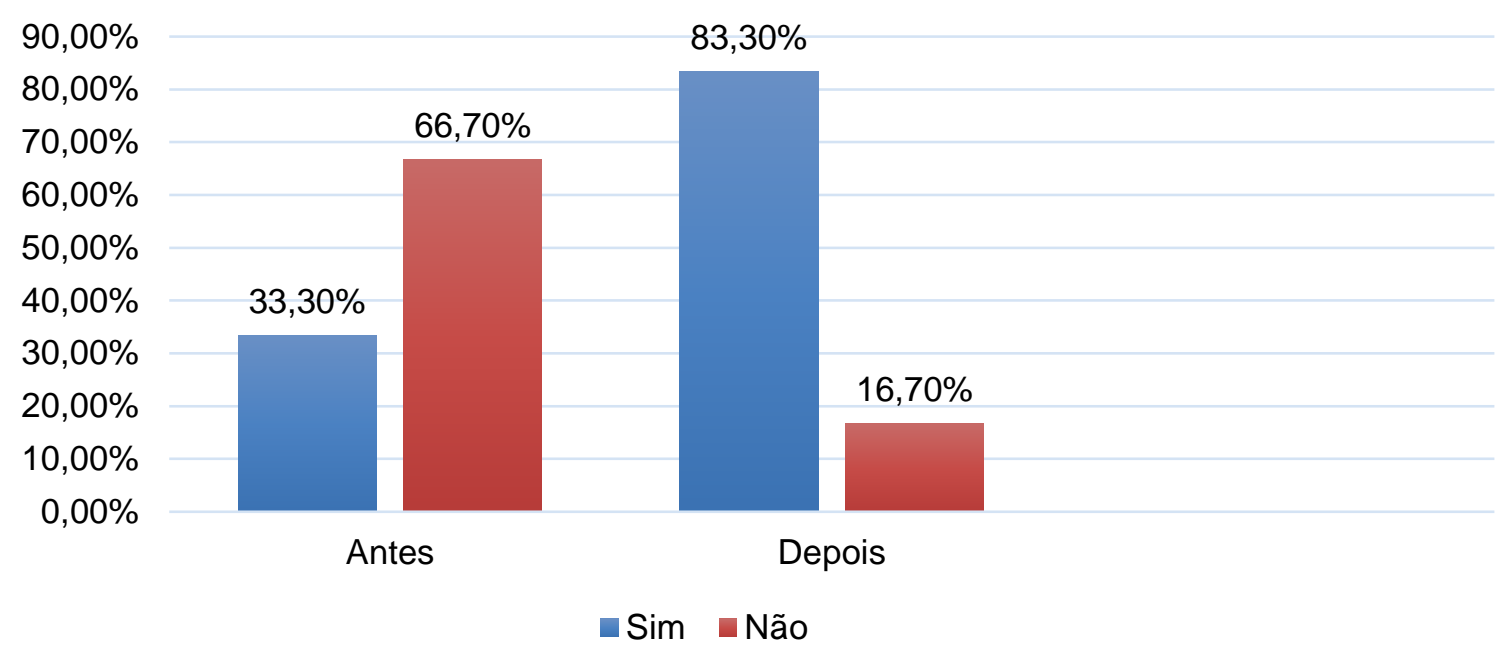

Fonte: Vasconcelos AM, et al., 2020.

A respeito do impacto na qualidade de vida dos idosos com depressão e o conhecimento dos ACS sobre o tema, antes da capacitação, $41,60 \%$ afirmaram reconhecer esse impacto negativo, enquanto $58,40 \%$ negaram esse conhecimento. Após a explanação dos pesquisadores, todos os pesquisados reconheceram os impactos causados pela depressão (Gráfico 4).

Gráfico 4 - Comparação das repostas acerca do conhecimento sobre os impactos da depressão na qualidade de vida dos idosos, pelos ACS, antes e depois da capacitação, na USF Panorama XXI, Belém - PA, Brasil, 2019.

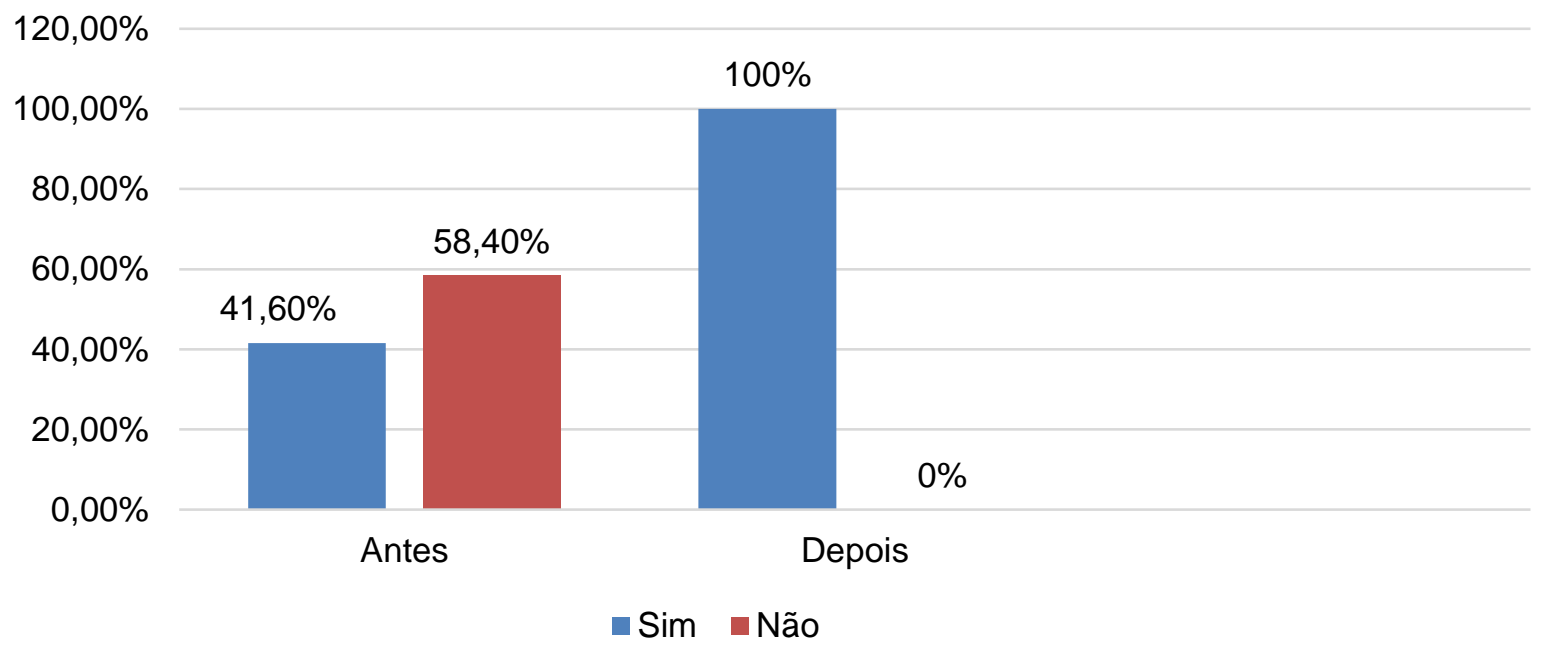

Fonte: Vasconcelos AM, et al., 2020. 
Quando os ACS foram questionados sobre outras oficinas e capacitações acerca da saúde mental na ESF do Panorama XXI, antes da capacitação, $91,70 \%$ dos pesquisados afirmaram que nunca houve essa abordagem na ESF em questão e 8,30\% afirmaram o contrário, enquanto que após a exposição sobre o tema, todos demonstraram como satisfatória a educação em saúde proposta pelos pesquisadores (Gráfico 5).

Gráfico 5 - Comparação das repostas acerca da participação em oficinas e capacitações realizadas na unidade que abordassem a saúde mental e a GDS, pelos ACS, antes e depois da capacitação, na USF Panorama XXI, Belém - PA, Brasil, 2019.

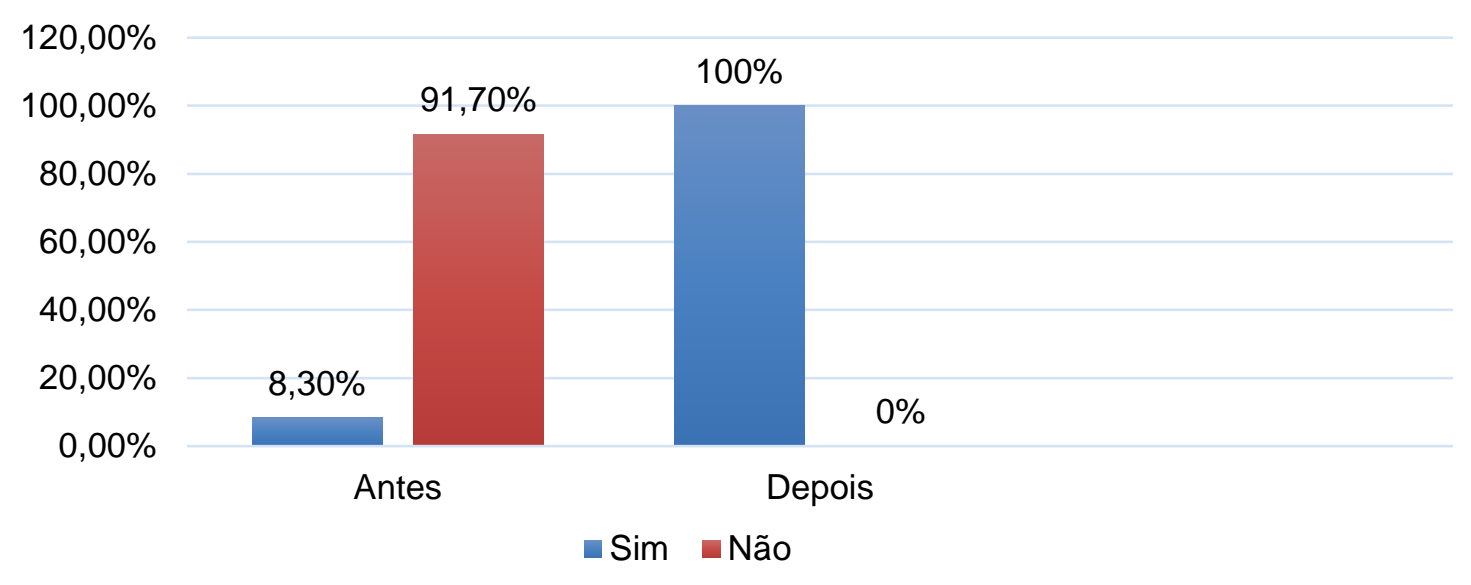

Fonte: Vasconcelos AM, et al., 2020.

\section{DISCUSSÃO}

Ao correlacionar todos os dados analisados para construção desta pesquisa, com os resultados encontrados anteriormente e posteriormente à realização da capacitação pelos pesquisadores, foi notória a grande variedade de fatores que levaram a necessidade de realização desse estudo. Destarte, podem ser elencados, principalmente, a falta de conhecimento dos ACS a respeito da Escala de Depressão Geriátrica GDS, a ausência de contato prévio destes com a GDS e, por fim, a falta de conhecimento a respeito da depressão em uma população da terceira idade, além do desconhecimento das suas consequências e da forma adequada de manejo por parte dos profissionais da saúde (ALMEIDA AM, et al., 2016).

À vista disso, as dificuldades dos ACS em relação à capacitação incipiente, podem ser devido à inserção deles no trabalho sem experiência anterior, à falta de projetos e/ou palestras que visem a qualificação desse profissional e com o agravante da falta de entendimento dos gestores no que diz respeito à importância das intervenções praticadas pelos ACS. Pois, para que o ACS realize de forma eficaz e concreta o seu trabalho, é preciso que ele receba uma qualificação suficiente para realizar suas diversas atribuições (SCHAAB BL, et al., 2017; ALMEIDA AM, et al., 2016).

De tal forma, foi percebido pelos pesquisadores que, ao haver um conhecimento adequado, por parte dos ACS - os quais têm papel preponderante na ligação entre paciente e atenção primária em saúde - a respeito da Escala GDS, bem como da Depressão em idosos, o suporte oferecido à esta população seria essencial para um bom prognóstico envolvendo esse transtorno psiquiátrico, além da melhoria da qualidade de vida desta população. Certamente, os ACS não terão respostas a todos os questionamentos feitos pela população, mas saberão direcionar os problemas que surgirem e dar um retorno à comunidade - medidas que fazem parte, inclusive, da credibilidade e aceitação que esse profissional tanto precisa para desenvolver sua rotina (ALMEIDA AM, et al., 2016). Tendo em vista a prevalência de transtornos psiquiátricos, principalmente depressão, em idosos, diversos estudos demonstraram que a GDS possui medidas válidas e confiáveis para a detecção de tal patologia. Sendo assim, sabe-se que é de extrema significância que a depressão na terceira idade seja identificada precocemente já que, de tal forma, se o seguimento correto for obtido, será relevante 
a melhora da qualidade de vida do paciente diagnosticado com esse transtorno, assegurando, dessa maneira, um envelhecimento ativo e a promoção da saúde mental da população idosa (KNOCHENHAUER CCLS e VIANNA KMP, 2016).

Como fora mencionado, sabe-se que o ACS é uma importante ferramenta da Atenção Primária em Saúde, sendo este o principal responsável pela ligação entre as famílias e o serviço de saúde; realizando o mapeamento de cada área, o cadastramento das famílias e estimulando a comunidade. Dessa maneira, percebeu-se a notoriedade de uma preparação adequada dos ACS a respeito do funcionamento, bem como, importância da GDS e sua aplicação, para a promoção e prevenção da saúde mental em uma população da terceira idade, contribuindo, assim, para a melhoria da qualidade de vida destes (BORTOLI FR, et al., 2017; NABBE P, et al., 2017).

Após a realização da capacitação, os pesquisadores questionaram aos ACS a respeito da efetividade da intervenção, indagando primariamente, se estes compreenderam a importância da GDS e em seguida, se os ACS se sentiam capacitados para a aplicação dela, obtendo resposta positiva de todos os participantes da pesquisa para ambas as perguntas. Por conseguinte, foi questionado se os pesquisados consideram útil a tabela para triagem de depressão dos idosos em uma comunidade. Como resposta para todos os questionamentos, foi percebido que houve satisfação por parte dos ACS, o que despertou ávido interesse deles acerca do assunto.

Isto posto, percebe-se com o resultado da pesquisa, que há uma equivalência entre o que foi encontrado e os estudos analisados. Comparando de forma horizontal as ferramentas do estudo proposto no referido trabalho com artigos fornecidos em plataforma virtual, pôde-se ratificar as dificuldades que se fazem presentes na rotina diária dos ACS, a necessidade de capacitações frequentes e a importância da interação da díade equipe-usuário, além da importância e eficácia da escala GDS (SCHAAB BL, et al., 2017; ALMEIDA AM, et al., 2016; PEDREIRA RBS, et al., 2016; MOREIRA CS, 2017; KNOCHENHAUER CCLS, VIANNA KMP. 2016; GOMES RCM, et al., 2015; BORTOLI FR, et al., 2017).

Por fim, fora realizada uma pequena simulação, envolvendo a aplicação da escala e, a partir daí, surgiram indagações, por parte dos participantes da pesquisa sobre a forma de abordagem do paciente. Os pesquisadores, por sua vez, sanaram todas as dúvidas destes, fazendo com que os ACS se sentissem totalmente contemplados sobre seus questionamentos.

\section{CONCLUSÃO}

Diante dos resultados obtidos, pode-se concluir que a atividade desenvolvida alcançou o efeito almejado, uma vez que os ACS demonstraram aquisição de novos conhecimentos e habilidades. Outro fator importante foi o relato dos ACS de que, a partir daquele momento, sentiam-se confiantes para, quando necessário, abordar um assunto tão importante. Aprenderam não somente como aplicar a GDS, mas também a interpretar seus resultados e como proceder quando concluída sua aplicação. Por fim, com a realização deste trabalho de intervenção, concluiu-se ser válido a utilização de estratégias que possibilitem aos ACS atuarem como elo entre os idosos com indícios de depressão e o enfrentamento desta temática. Estratégias de avaliação rápida, como a GDS, permitem examinar uma grande parcela da população-alvo, facilitando a referência para os serviços médicos existentes. Desta forma, imprime-se a necessidade de qualificação permanente dos ACS, incorporando novos elementos à prática e aos conceitos prévios, como maneira de transformação da realidade.

\section{REFERÊNCIAS}

1. ALMEIDA AM, et al. Dificuldades dos agentes comunitários de saúde na prática diária. Rev Med Minas Gerais 2016; $26: e-1800$.

2. BORTOLI FR, et al. Conhecimento dos Agentes Comunitários de Saúde em relação à saúde bucal. Sau. \&Transf. Soc., ISSN 2178-7085, Florianópolis, v.8, n.2, mai-ago, p.96-102, 2017. 
3. FIGUEIREDO EN. A Estratégia Saúde da Família na Atenção Básica do SUS. Especialização em Saúde da Família. UNA-SUS | UNIFESP. Unid 05. 2015.

4. GALERA SC, et al. Educação Médica em Geriatria: Desafio Brasileiro e Mundial. Geriatr., Gerontol. Aging (Impr.); 11(2): 88-94, abr.-jun. 2017.

5. GOMES RCM, et al. O trabalho do agente comunitário de saúde na perspectiva da educação popular em saúde: possibilidades e desafios. The work of the community health worker from the perspective of popular health education: possibilities and challenges, 2015.

6. GONZÁLEZ ACT, et al. Transtornos depressivos e algumas comorbidades em idosos: um estudo de base populacional. Rev. Bras. Geriatr. Gerontol., Rio de Janeiro, 2016; 19(1):95-103.

7. KNOCHENHAUER CCLS, VIANNA KMP. Percepção dos Agentes Comunitários de Saúde quanto aos agravos fonoaudiológicos. Community Health Workers perceptions in relation to speech and language disorders. CoDAS 2016;28(6):697-703.

8. LAUDISIO A, et al. Definition of a Geriatric Depression Scale cutoff based uponquality of life: a population-based study. Int J Geriatr Psychiatry. 2017 Mar 31.

9. LIMA AMP, et al. Depressão em idosos: uma revisão sistemática da literatura. R Epidemiol Control Infec, Santa Cruz do Sul, 6(2):97-103, 2016. [ISSN 2238-3360].

10. MOREIRA CS. Projeto de intervenção para capacitação de agentes comunitários de saúde em uma estratégia de saúde da família. UFMG, Curso de Especialização em Saúde da Família, 2017.

11. NABBE $P$, et al. Which DSM validated tools for diagnosing depression are usable in primary care research? A systematic literature review. European Psychiatry. Volume 39, 2017, Pages 99-105.

12. PEDREIRA RBS, et al. Validade de conteúdo do Instrumento de Avaliação da Saúde do Idoso. einstein. 2016;14(2):158-77.

13. RALDI GV, et al. Avaliação da prevalência de depressão em idosos institucionalizados em uma ILPI no Norte do RS. Revista de Enfermagem | FW | v. 12 | n. 12 | p.48-63 | 2016.

14. RAMOS GCF, et al. Prevalência de sintomas depressivos e fatores associados em idosos no norte de Minas Gerais: um estudo de base populacional. J Bras Psiquiatr. 2015;64(2):122-31.

15. SANTOS-ORLANDI AA, et al. Elderly who take care of elderly: a study on the Frailty Syndrome. Rev Bras Enferm [Internet]. 2017;70(7):822-9.

16. SCHAAB BL, et al. Estudos para construção e propriedades psicométricas da escala contextual de depressão em idosos. Instituto Metodista de Ensino Superior CGC. 2017:25(1).

17. SEMED DC, et al. Fatores Associados a Depressão e os Cuidados de Enfermagem no Idoso. Revista de Enfermagem | FW | v. 12 | n. 12 | p.101-113 | 2016.

18. SILVA AR, et al. Doenças crônicas não transmissíveis e fatores sociodemográficos associados a sintomas de depressão em idosos. J. bras. psiquiatr. [Internet]. 2017; 66 (1): 45-51. 